\title{
Gestión global de talento en una empresa multinacional del sector de la automoción
}

\author{
Marçal Vázquez \\ Departamento de Producción, Grupo Volkswagen, China \\ marcial.vazquez@faw-vw.com
}

\author{
Albert Sunyer \\ Universitat Politècnica de Catalunya \\ albert.sunyer.torrents@upc.edu
}

Resumen. La gestión del talento abarca la selección, contratación, incorporación, desarrollo, retención y renovación de recursos bumanos, teniendo en cuenta el análisis y la planificación global de estos. Atraer y retener el talento adecuado en los puestos de trabajo es uno de los factores clave del éxito de las empresas en el competitivo panorama de la economía global (Collings y Mellabi, 2009).

El objetivo de este estudio es mostrar el proceso de gestión global de talento de una empresa a través de las diferentes fases que lo componen: detección, desarrollo, evaluación y confirmación. Para ello, esta investigación utiliza una metodología basada en el estudio de un caso en profundidad a partir del análisis de una empresa dedicada al desarrollo, fabricación y distribución logística de automóviles.

Los resultados de esta investigación muestran que la empresa analizada gestiona el talento de forma global y sistemática (Tarique y Schuler, 2010), generando un pool de talento, ofreciendo múltiples vías de desarrollo profesional y fomentando de igual manera la movilidad intra- e interárea a través de observaciones por múltiples evaluadores (directivos tanto de la propia área del candidato como de otras áreas). La metodología desarrollada por la empresa es innovadora en tres aspectos: 1) la autonominación, 2) la evaluación on the job, y 3) la evaluación por múltiples evaluadores. Palabras clave: programas de desarrollo; gestión de personal; promoción del personal; carrera profesional; evaluación y rendimiento de personal; personal directivo. 
Global Talent Management in a Multinational Company in the Automotive Sector

Abstract. Talent management involves the selection, recruitment, onboarding, development, retention, renewal of analysis and overall planning buman resources. To this end, attracting and retaining the right talent are key factors for ensuring company success in the competitive landscape of the global economy (Collings and Mellabi, 2009).

The aim of this study is to show the overall talent management process of a company through the different phases of detection, development, assessment and confirmation. To this end, this research uses an in-depth case study approach based on the analysis of a company that works in the development, manufacture and logistic distribution of automotive vebicles.

The results show that the company has understood the importance of managing talent in a global and systematic way (Tarique and Schuler, 2010) because it has generated a talent pool, offered multiple ways of professional development and promoted intra-and inter-area mobility based on observations by multiple evaluators (managers from the candidate's area and other areas). The methodology implemented by the company is innovative in three areas: 1) self-nomination, 2) on the job evaluation, and 3) assessment by multiple evaluators.

Keywords: development program; buman resources management; promotion; professional career; performance assessment; executive development. 


\section{Introducción}

La investigación en dirección de empresas muestra un creciente interés por la gestión del talento internacional como una herramienta competitiva (Farndale, Scullion y Sparrow, 2010). Asimismo, las organizaciones empresariales señalan una gran dificultad para detectar y evaluar el talento con precisión, lo que refleja la falta de fundamento teórico para la identificación y el desarrollo de este (Nijs, Gallardo, Dries y Sels, 2014). Diversos autores indican que, pese al gran interés mostrado en la gestión del talento en las empresas, todavía existe un distanciamiento considerable entre el ámbito académico y el profesional, con serias lagunas académico-prácticas (Rynes, Giluk y Brown, 2007). El presente estudio pretende reducir esta brecha, mostrando cómo el Departamento de Recursos Humanos de una empresa dedicada al sector de la automoción, con una continua y creciente cultura competitiva, ha implantado una metodología para la gestión global del talento.

Para la realización de esta investigación se ha utilizado el estudio de un caso en profundidad y se ha analizado el proceso de gestión global del talento en la empresa seleccionada. Para ello se ha recogido información procedente de documentación incluida en los procedimientos internos de la empresa, la participación directa de uno de los autores como observador en el proceso de gestión global del talento y la realización de 35 entrevistas semiestructuradas a directivos de diferentes áreas de la empresa.

Partiendo de investigaciones previas, este estudio aporta nuevos conocimientos acerca de la implantación de un proceso sistemático de gestión global de talento, describiendo la metodología empleada por el Departamento de Recursos Humanos para la detección, desarrollo, visibilidad-evaluación y confirmación del talento. Adicionalmente, se han identificado una serie de factores innovadores que complementan los estudios previos sobre la gestión global del talento.

\section{Revisión de la literatura}

\subsection{La gestión del talento en las organizaciones}

La palabra talento surgió como una unidad de conversión a moneda de cambio, teniendo un valor medible a través del peso $(1$ talento $=25,86 \mathrm{~kg})$. Sus orígenes se remontan a la antigua Grecia a través de la palabra tálanton, que posteriormente se trasladó al latín talentum (Givhan, 2014). Sin embargo, fue durante el siglo xIx cuando la palabra talento tuvo por primera vez un significado ligado a la persona (sujeto) en lugar de un enfoque hacia las características de una persona (objeto) (Tansley, 2011). 
El significado de la palabra talento se ha debatido intensamente en la investigación sobre dirección de empresas (Gallardo, Dries y González-Cruz, 2013). Tansley, Turner y Foster (2007) definieron como talentos a aquellos empleados de los que se espera que marquen una diferencia en el rendimiento de la organización mediante su contribución inmediata o su expectativa de rendimiento a largo plazo. Es por ello que el talento en el contexto empresarial se refiere a las personas que demuestran regularmente una habilidad y un logro excepcional, ya sea en una gama de actividades y situaciones o dentro de un campo especializado, donde se muestre sistemáticamente una alta capacidad en situaciones donde los conocimientos no sean altamente aplicables (Williams, 2000). Paralelamente, también se considera talentos a los empleados de alto rendimiento y/o de alto potencial que se tienen en cuenta para ser los futuros líderes de la empresa (Björkman, Ehrnrooth, Mäkelä, Smale y Sumelius, 2013; Mäkelä, Björkman y Ehrnrooth, 2010).

Los orígenes de la gestión del talento en el contexto empresarial se remontan a la década de los noventa con el estudio titulado The War for Talent (Michaels, Handfield-Jones y Axelrod, 2001). La gestión del talento abarca los procesos, la administración y las tecnologías de recursos humanos. Comúnmente se refiere a la contratación, selección, incorporación, retención, desarrollo y renovación de los recursos humanos, teniendo en cuenta el análisis y la planificación global de estos. En otras palabras, la gestión del talento es lo que ocurre en el nexo de los procesos de contratación, desarrollo y gestión de la fuerza de trabajo y puede describirse alternativamente como la optimización del talento (Schweyer, 2004). Sin embargo, pese al creciente interés de la investigación científica en la gestión del talento, algunas preguntas básicas están todavía sin responder, como son, por ejemplo: ¿cómo afecta el talento al rendimiento, competencia o el compromiso del individuo?, ¿es el talento una habilidad natural o se adquiere a través de la enseñanza teórica y práctica?, ¿cómo puede gestionarse el talento de forma sistemática en las empresas? (Gallardo et alii, 2013).

\subsection{Gestión global del talento (global talent management o GTM)}

Existe un consenso cada vez mayor en cuanto a que la gestión global del talento es un área emergente en las empresas. Sin embargo, no hay consenso en cuanto a la definición exacta del concepto en cuestión, sino que más bien existe un considerable debate en torno a la definición del término (Collings y Mellahi, 2009; Lewis y Heckman, 2006; Tarique y Schuler, 2010). La gestión global del talento está orientada al futuro y se enmarca en la planificación de RR. HH. con la proyección de las necesidades de empleados o personal a nivel internacional. Aquí el enfo- 
que está en los tipos de capacidades profesionales que se necesitarán en el futuro, centrándose en los individuos de alto rendimiento, también conocidos como de alto potencial. Asimismo, se orienta a los puestos de trabajo estratégicos, que son fundamentales para la organización en términos de creación de ventaja competitiva para las empresas. En definitiva, se trata de «un subconjunto de actividades sistemáticas de internacionalización de la gestión de RR. HH. para atraer, desarrollar, retener y movilizar a personas con altos niveles de capital humano actual y potencial, coherente con los objetivos estratégicos de las empresas multinacionales en un entorno dinámico, global y altamente competitivo» (ibid., 124).

Independientemente de la definición que se utilice, hay un tema común en todas ellas. Parece que la gestión global del talento se centra en dos dimensiones principales: individuos con altos niveles de competencias (por ejemplo, conocimientos, habilidades y destrezas) que agregan valor a la organización y políticas o prácticas internacionales complementarias de dirección de RR. HH. que se utilizan para gestionar a los empleados con niveles de competencia elevados.

El estudio de Arkless (2011) recomienda adoptar un enfoque sistemático para reducir los riesgos en la gestión global del talento, sugiriendo varias propuestas a las empresas multinacionales como son introducir la planificación estratégica de la fuerza de trabajo (por ejemplo, determinar las futuras brechas de talento), facilitar la migración (por ejemplo, atraer el talento mundial), fomentar la circulación de know-how (por ejemplo, reducir la fuga de talento facilitando a los estudiantes y profesionales el regreso a su país de origen), crear un pool (o reserva) de talento (por ejemplo, ofreciendo múltiples vías de desarrollo y de carrera profesional), o ampliar el pool de talento (por ejemplo, atrayendo a colectivos como mujeres, profesionales mayores, etc.). De este modo, la gestión global del talento en las empresas multinacionales incluiría «todas las actividades organizativas con el fin de atraer, seleccionar, desarrollar y retener a los mejores empleados en las funciones más estratégicas a escala mundial» (Scullion, Collings y Caligiuri, 2010).

Por lo anteriormente descrito, la gestión global del talento es un campo en auge que se centra en la optimización de los recursos humanos de las empresas internacionales para proporcionar una ventaja competitiva sostenida (por ejemplo, a través de planes de carrera), aunque permanece, en gran medida, desconectada de la gestión diaria del departamento de RR. HH. (Crowley, Benson y Akram, 2019). 


\subsection{Fases del proceso GTM}

Una de las actividades principales en cualquier organización es el desarrollo y retención de los mejores trabajadores (Farndale et alii, 2010; McDonnell, Lamare, Gunnigle y Lavelle, 2010; Stahl et alii, 2012). Para ello, atraer y retener el talento es uno de los factores clave del éxito de las empresas en el entorno competitivo de la economía global (Collings y Mellahi, 2009).

Una herramienta extendida entre las empresas internacionales consiste en clasificar a los trabajadores identificados como talento (Boudreau y Ramstad, 2005), creando diferentes pools (o reservas) de talento de acuerdo con las etapas de la carrera profesional en la que esos individuos se encuentren (Mäkelä et alii, 2010). Los pools de talento pueden estar segmentados, por ejemplo, como «alto potencial en los comienzos de la carrera profesional», «especialistas»o «altos ejecutivos» (Stahl et alii, 2007).

El proceso de gestión del talento suele incluir varias fases: identificación (a quién desarrollar), diseño (qué competencias desarrollar, a qué velocidad y en cuánto tiempo), evaluación (qué instrumentos analíticos utilizar para medir la eficacia) y apoyo organizativo (qué apoyo puede prestar la alta dirección) (Garavan, Carbery y Rock, 2012).

\subsubsection{Fase de identificación y selección}

Las organizaciones deberían prestar una atención especial en la cuestión de cómo identificar y seleccionar el talento, empezando por analizar cuáles son las características que debe tener una persona (externa o interna a la empresa) para que esta sea considerada talento (Björkman et alii, 2013). El papel de la planificación de los RR. HH. para identificar las necesidades futuras es importante en todos los niveles de la organización (Cheese, 2010; Frauenheim, 2009; Freedman, 2009; Harttig, 2010; Hills, 2009; Lawler, 2008). De modo que la fase de identificación del talento es necesaria para que las empresas sean más eficientes a la hora de invertir los recursos de selección (Colombo, 2006; Naim, 2014).

Externamente, las empresas pueden identificar $y$ atraer a candidatos utilizando los métodos de selección de personal o de head bunting (caza de talento) (Vasile y Zhan, 2020). En la selección de personal se publicita una oferta de empleo y los seleccionadores esperan que los candidatos se interesen en esa posición, mientras que en el head bunting las empresas de consultoría contactan directamente con posibles candidatos que cumplan con los requisitos del puesto, siendo el contacto directo cada vez más accesible a través de redes sociales como LinkedIn (Chen, Lee y Ting, 2017). Internamente, las organizaciones pueden utilizar eficazmente las prácticas de gestión del desempeño para identificar el talento y 
rediseñar los puestos de trabajo o reestructurar el trabajo con el objetivo de motivar e involucrar más a los empleados (Guthridge, McPherson y Wolf, 2009). En definitiva, la productividad y el alto rendimiento de una organización dependen de la correcta selección de la persona, así como ubicarla en el puesto de trabajo indicado para él/ella (Huselid, 1995). Para que la selección del candidato sea efectiva, también es importante definir con la mayor precisión posible el puesto de trabajo que cubrir (Byars y Rue, 1994).

En la literatura sobre gestión del talento se supone que la mayoría de las personas con talento son los propios empleados de la empresa (Tarique y Schuler, 2010), por lo que un reto para las organizaciones es cómo llegar a crear esta reserva de talento. Es común que las empresas interesadas en la identificación del talento hayan creado grupos de trabajo denominados círculos de talento o pools de talento, los cuales están integrados por personal con ciertas habilidades que les caracterizan para ser futuros directivos de la organización (Xuy et alii, 2016). Las políticas de selección del talento a nivel mundial suelen seguir una estrategia de reserva de talento, lo que implica contratar a los individuos con más talento y luego colocarlos en posiciones clave, colaborar con centros generadores de talento como universidades y escuelas de negocio y ser muy selectivos en la contratación de nuevos empleados (Stahl et alii, 2012).

\subsubsection{Fase de desarrollo}

Un proceso de desarrollo profesional adecuado para los empleados permite ampliar sus conocimientos y habilidades, los cuales son la base para la evaluación de sus capacidades como potencial en la empresa (Fernández-Aráoz, Groysberg y Nohria, 2011). El desarrollo profesional incluye la adquisición y desarrollo sistemático de los conocimientos, habilidades y aptitudes requeridos para cada puesto de trabajo con el objetivo de que los empleados mejoren el desempeño de las tareas que ya realizan o bien para mejorar su rendimiento en el entorno laboral (Goldstein, 1990; Latham, Saari, Pursell y Campion, 1980).

La capacitación y el desarrollo de los trabajadores identificados como talento abarcan la promoción interna, la evaluación continua de las necesidades de formación y otras prácticas de gestión de la trayectoria profesional (Bonneton, Festing, Schworm y Muratbekova-Touron, 2019), como son las asignaciones internacionales a corto y largo plazo, la participación en equipos globales, la capacitación intercultural, la asignación de mentores y los programas de formación (Caligiuri y Tarique, 2009). El desarrollo de políticas de talento suele incluir el desarrollo del liderazgo, la promoción dentro de la empresa y el desarrollo individual alineado con los planes de sucesión (Stahl et alii, 2012). 


\subsubsection{Fase de retención}

La retención de empleados implica evitar su pérdida (Sheridan, 1992). En particular, es difícil mantener el talento puesto que estos empleados son los primeros en plantearse abandonar la organización dado que tienen más opciones en el mundo laboral exterior (Bradberry, 2016). Por ello, las empresas que trabajen en el diseño de una estrategia futura sostenible deben incluir en la hoja de ruta de los RR. HH. la retención del talento y su compromiso con la organización (Sylvester, 2004).

Según Mukhtar et alii (2011), un tercio de los mejores trabajadores no se sienten a gusto con su jefe y están buscando un nuevo puesto de trabajo continuamente. Ocurre que poco a poco van perdiendo el interés y la pasión por su trabajo, un fenómeno llamado caída de tensión. Según este fenómeno, los trabajadores con talento ofrecen un buen rendimiento en su trabajo, contribuyendo y trabajando en equipo, pero de forma sorprendente pueden plantearse su marcha de la empresa.

La retención del talento se refiere a las políticas y prácticas por parte de la organización (generalmente en el área de los RR. HH.) para prevenir anticipadamente su fuga. Consiste en tomar medidas para animar a los empleados a permanecer dentro de la organización (Björkman et alii, 2013). La gestión de esta retención engloba un conjunto de prácticas de RR. HH. que las organizaciones desarrollan para reducir las tasas de rotación voluntaria, puesto que la rotación de los empleados provoca fugas de conocimiento organizativo y la sustitución de estos es costosa tanto en términos económicos como en plazos (DeVos y Meganck, 2007). La gestión de la retención incluye, por ejemplo, la atención del personal directivo hacia los empleados, así como las prácticas de conciliación entre la vida laboral y personal (Bonneton et alii, 2019). Las políticas de retención del talento suelen incluir la creación de planes de carrera personalizados, acuerdos de trabajo flexible y la atención a los niveles de disminución del rendimiento (Stahl et alii, 2012).

\section{Metodología}

\subsection{Contexto de la investigación}

Esta investigación se basa en el estudio de un caso en profundidad sobre la gestión global del talento (Eisenhardt, 1989; Eisenhardt y Graebner, 2007; Yin, 2011). La metodología de estudio de casos en profundidad ya ha sido empleada de forma similar en diferentes investigaciones sobre la gestión del talento por autores como Iles, Chuai y Preece (2010) o Bonneton et alii (2019). Los motivos que han conducido a la elección de esta metodología son la complejidad y la sin- 
gularidad del proceso de gestión global del talento. A través del análisis del caso en cuestión, se pretende obtener una descripción detallada del proceso, así como los agentes que intervienen y los resultados obtenidos por la empresa (Gibbert y Ruigrok, 2010).

La actividad de la empresa escogida es el desarrollo, fabricación y distribución logística de automóviles. La empresa forma parte de una corporación con un total de 14 marcas procedentes de 7 países europeos y que en su conjunto forman uno de los grupos automovilísticos más importantes a nivel mundial con presencia en más de 150 países. La sede corporativa se ubica en Europa, contando con un total de más de 650.000 empleados. Esta empresa se ha escogido por su dimensión, competitividad y políticas de gestión global del talento, lo que le ha supuesto haber obtenido durante 6 años consecutivos ser empresa Top Employer ${ }^{1}$ en España, una distinción que evalúa a las empresas que ofrecen mejores condiciones de trabajo y resultan más atractivas para los trabajadores a nivel mundial.

\subsection{Recogida de datos}

El estudio realizado se basa en tres fuentes complementarias de datos primarios: 1) entrevistas realizadas a 35 directivos involucrados en el programa de gestión global del talento de la empresa estudiada, ya sea en el papel de observadores, candidatos o gestores del programa en el departamento de RR. HH. de la empresa, 2) observación participante por parte de uno de los autores, que ha participado como evaluador y persona de contraste en el programa de gestión global del talento, y 3) documentación interna facilitada por RR. HH. relativa al programa de gestión global de talento de la empresa.

La tabla 1 muestra el perfil de los participantes, indicando su cargo, área funcional, sexo, nacionalidad, edad y antigüedad en la empresa. Las 35 entrevistas semiestructuradas fueron la principal fuente de datos primarios, tuvieron una duración de entre 30 y 60 minutos y se realizaron sistemáticamente hasta alcanzar la saturación de los datos (Guest, Bunce y Johnson, 2006). Algunas de las preguntas de las entrevistas incluyeron la participación del entrevistado en el programa de gestión de talento, su conocimiento o experiencia sobre el programa, cómo potenciar el talento de la empresa, cómo detectar el talento de la empresa, qué valores deben tener los futuros directivos de la empresa, cómo ha evolucionado el programa de gestión de talento en los últimos años, etc. Las entrevistas fueron transcritas e introducidas en el programa de investigación cualitativa Atlas.ti para su posterior codificación y análisis junto con el resto de documentos recopilados.

1 www.top-employers.com 
Tabla 1. Perfil de las personas entrevistadas Variables

\begin{tabular}{|c|c|c|c|}
\hline & & $\%$ & Individuos \\
\hline \multirow{7}{*}{ Cargo } & Presidencia & $2,86 \%$ & 1 \\
\hline & Vicepresidencia & $2,86 \%$ & 1 \\
\hline & Director & $20,00 \%$ & 7 \\
\hline & Gerente & $28,57 \%$ & 10 \\
\hline & Mánager & $17,14 \%$ & 6 \\
\hline & Extraconvenio & $22,86 \%$ & 8 \\
\hline & Convenio & $5,71 \%$ & 2 \\
\hline \multirow{8}{*}{ Área funcional } & Producción & $37,14 \%$ & 13 \\
\hline & Calidad & $25,71 \%$ & 9 \\
\hline & Recursos Humanos & $11,43 \%$ & 4 \\
\hline & $\mathrm{I}+\mathrm{D}$ & $8,57 \%$ & 3 \\
\hline & Finanzas & $8,57 \%$ & 3 \\
\hline & Logística & $2,86 \%$ & 1 \\
\hline & Tecnol. de Información & $2,86 \%$ & 1 \\
\hline & Presidencia & $2,86 \%$ & 1 \\
\hline \multirow{2}{*}{ Sexo } & Hombre & $82,86 \%$ & 29 \\
\hline & Mujer & $17,14 \%$ & 6 \\
\hline \multirow{3}{*}{ Nacionalidad } & Española & $80,00 \%$ & 28 \\
\hline & Alemana & $14,29 \%$ & 5 \\
\hline & Otras & $5,71 \%$ & 2 \\
\hline \multirow{5}{*}{ Edad } & $<30$ & $0,00 \%$ & 0 \\
\hline & $30-39$ & $28,57 \%$ & 10 \\
\hline & $40-49$ & $28,57 \%$ & 10 \\
\hline & $50-59$ & $42,86 \%$ & 15 \\
\hline & $>59$ & $0,00 \%$ & 0 \\
\hline \multirow{7}{*}{ Antigüedad } & $<5$ & $20,00 \%$ & 7 \\
\hline & $5-9$ & $11,43 \%$ & 4 \\
\hline & $10-14$ & $11,43 \%$ & 4 \\
\hline & $15-19$ & $11,43 \%$ & 4 \\
\hline & $20-24$ & $2,86 \%$ & 1 \\
\hline & $25-29$ & $31,43 \%$ & 11 \\
\hline & $>29$ & $11,43 \%$ & 4 \\
\hline
\end{tabular}




\subsection{Análisis de datos cualitativos}

El proceso inductivo se ha basado en el análisis temático del discurso (Riessman, 2005; Crossley, 2007), siguiendo el método reduccionista mediante la comparación y contraste de los datos recopilados (Collis y Hussey, 2009). Ambos autores analizaron los datos recogidos para profundizar en la comprensión del proceso de gestión global del talento en la empresa seleccionada. Este análisis permitió entender la complejidad y singularidad de las distintas fases del proceso al mismo tiempo que evitar cualquier tipo de sesgo (Miles y Huberman, 1994). Se prestó especial atención en la descripción de los objetivos, las tareas y los participantes involucrados en cada fase del proceso. Se identificaron 20 códigos clave que emergieron de la lectura de los textos que fueron utilizados como elementos narrativos de primer orden. Estos códigos fueron agrupados en 4 temas más amplios que permitieron observar el flujo lógico en la progresión de los eventos y de las actividades desarrolladas (Tansey, 2007). La tabla 2 muestra la estructura y agrupación de los códigos utilizados. Finalmente, se crearon mapas visuales con las conexiones más importantes entre los temas para identificar patrones y organizar las relaciones existentes entre ellas (ver figura 1). El siguiente apartado muestra los resultados obtenidos en este análisis y los patrones observados.

Tabla 2. Estructura de códigos y su fundamentación en el análisis cualitativo

\begin{tabular}{|l|l|c|}
\hline \multirow{4}{*}{ Grupo (tema) } & Códigos de primer orden & $\begin{array}{c}\text { Citas en la } \\
\text { base de datos }\end{array}$ \\
\hline \multirow{4}{*}{ 01 DETECTAR TALENTO } & Autonominación & 3 \\
\cline { 2 - 3 } & Autorresponsabilidad & 8 \\
\cline { 2 - 3 } & Dar oportunidad a la persona & 9 \\
\cline { 2 - 3 } & Nominación vía superior & 7 \\
\cline { 2 - 3 } TALENTO & Observación del talento & 11 \\
\cline { 2 - 3 } & Proceso de selección & 4 \\
\hline \multirow{5}{*}{ DESARROLLAR EL } & Desarrollar competencias & 7 \\
\cline { 2 - 3 } & Desarrollar creatividad & 5 \\
\cline { 2 - 3 } & Desarrollar potencial & 4 \\
\cline { 2 - 3 } & Development day & 11 \\
\cline { 2 - 3 } & Programas de desarrollo & 5 \\
\cline { 2 - 3 } & Proponer retos & \\
\hline
\end{tabular}




\begin{tabular}{|l|l|c|}
\hline \multirow{4}{*}{ Grupo (tema) } & Códigos de primer orden & $\begin{array}{c}\text { Citas en la } \\
\text { base de datos }\end{array}$ \\
\hline \multirow{4}{*}{$\begin{array}{l}\text { 03 DAR VISIBILIDAD Y } \\
\text { EVALUAR EL TALENTO }\end{array}$} & Dar visibilidad a la persona & 8 \\
\cline { 2 - 3 } & Evaluación de competencias & 5 \\
\cline { 2 - 3 } & Evaluación on the job & 7 \\
\cline { 2 - 3 } & Proceso de evaluación & 4 \\
\hline \multirow{4}{*}{$\begin{array}{l}\text { 04 CONFIRMACIÓN DEL } \\
\text { TALENTO }\end{array}$} & Confirmación como mánager & 3 \\
\cline { 2 - 3 } & Feedback de confirmación & 5 \\
\cline { 2 - 3 } & Múltiples ojos & 5 \\
\cline { 2 - 3 } & Regular monitoring & 3 \\
\hline
\end{tabular}

\section{El programa de global talent management (GTM) en la empresa estudiada}

\subsection{Principios del programa GTM estudiado}

El programa GTM de la empresa estudiada está diseñado para reforzar el principio de evaluación a través de múltiples observadores, permitiendo, a su vez, la autorresponsabilidad, dado que en este proceso el candidato puede autonominarse para un puesto directivo. Posteriormente, múltiples observadores evalúan al candidato en situaciones cotidianas en el ámbito de su responsabilidad, promoviendo la diversidad y la transparencia.

Uno de los gerentes de mayor experiencia de la empresa es el Sr. Martí, quien matiza: «Considero que la detección y desarrollo del talento es uno de los apartados claves en cualquier ámbito empresarial». De igual manera, el Sr. González también indica: «Para detectar personas con talento podemos plantear retos a los candidatos que consideremos para ver cómo los resuelven, y nosotros como líderes tenemos que descubrirlos, desarrollarlos y hacer todo lo posible por potenciarlos».

En el programa GTM se evalúan una serie de competencias: integridad (autorresponsabilidad y autodesarrollo), trabajo en equipo, innovación (actuar estratégicamente) y orientación a los resultados (eficiencia en la complejidad). Iniciar este proceso depende del candidato, el cual debe buscar retos y responsabilidades con el fin de desarrollar las competencias y conseguir un buen rendimiento.

Un aspecto diferencial de este programa GTM es el cambio de perspectiva, sustituyendo el diagnóstico selectivo que se realizaba previamente en un assessment center fuera de la empresa, con entrevistas y role plays, por el diagnóstico de selección continuo mostrado y evaluado directamente en el ámbito de res- 
ponsabilidad que tiene el candidato en situaciones de rutina diaria (on the job) mostrando su comportamiento en situaciones reales.

Tal y como indica la Srta. Raquel (de 33 años y 7 de experiencia en la empresa): «Es un proceso de evaluación continua del personal con el que trabajas diariamente; cómo se desarrollan y actúan en el día a día; con qué motivaciones y expectativas, dándoles oportunidades que puedan hacer aumentar su motivación y que hagan que sientan que están creciendo, mitigar la sensación de estanqueidad o de haber llegado a un máximo».

El otro aspecto diferencial que ofrece el actual programa de GTM es la potenciación de la autorresponsabilidad iniciada en el proceso de autonominación como candidato del programa. Iniciar este proceso depende de los candidatos y de su interés en buscar nuevos retos y responsabilidades, desarrollar competencias y conseguir un buen resultado, tal y como se refleja en el manual del programa GTM de la empresa estudiada.

El principio de múltiples evaluadores permite obtener diferentes puntos de vista, aumentando considerablemente el conocimiento y la objetividad en la evaluación del candidato. Carlos (gerente de 46 años y amplia experiencia), hace hincapié en la detección del talento, reforzando la idea de que el candidato «debe pasar por el principio de múltiples ojos, como, por ejemplo, rondas comparativas. El talento se potencia con programas de desarrollo, lo que significa, bajo mi punto de vista, no solo formación, sino también proyectos multidisciplinares, interárea, etc.».

\subsection{Fases del programa GTM en la empresa estudiada}

\subsubsection{Fase 01. Detectar el talento}

En la fase inicial del programa GTM se abre un espacio de autorreflexión. Durante esta, el candidato debe reflexionar y realizar autocrítica sobre sus propias aptitudes, metas profesionales, intereses y competencias, y, con base en todo ello, tomar la decisión de iniciar o no el programa GTM.

Es de vital importancia que el candidato piense qué implica ser un cargo directivo en la empresa, reflexionar sobre las consecuencias e implicaciones del puesto, así como valorar su capacidad y motivación real.

Los principales objetivos durante esta fase son los siguientes:

1. Promover la reflexión personal sobre el propio desarrollo profesional.

2. Fomentar la retroalimentación al candidato por parte de su responsable y sus compañeros.

3. Poner a disposición del candidato los recursos de información necesarios para ayudarle a tomar la decisión. 
Puesto que la autorreflexión y la autorresponsabilidad son de suma importancia en esta fase, el sistema de e-HRM (sistema digital de recursos humanos) dispone de información y recursos que ayudan al candidato a conocerse mejor.

En esta fase es imprescindible que el candidato cumpla los requisitos de acceso para pasar a la siguiente fase con el objetivo de hacer un primer filtro de candidatos. Así pues, la empresa ha definido una serie de requisitos mínimos:

1. Experiencia profesional mínima, incluyendo otras empresas.

2. Antigüedad mínima en la empresa.

3. Conocimientos de inglés o alemán, nivel B2.

4. Evaluación anual favorable.

5. Posición de extraconvenio o convenio con responsabilidades adicionales.

En relación con la detección del talento, el Sr. Díaz (53 años y más de 27 de experiencia) expone: «El talento se detecta observando la facilidad que tiene una persona en realizar algo que para el resto es complicado»; también el Sr. Iglesias (gerente con más de 30 años en la empresa) indica: «Se detecta en el día a día observando cómo se desenvuelven en la gestión para la consecución de los objetivos».

\subsubsection{Fase 02. Desarrollar el talento}

Esta fase se centra en la evaluación del rendimiento y de la personalidad del candidato como talento potencial e incluye varias herramientas de evaluación y desarrollo.

La primera herramienta consiste en un análisis del perfil del candidato y aporta un informe detallado y un resultado final. El informe contiene información que el candidato puede emplear para conocerse mejor y buscar vías de desarrollo, permitiendo identificar sus fortalezas y áreas de mejora, contrastar su autopercepción frente a la percepción de los observadores, comprobar su nivel de competencias y ayudarle a reflexionar.

La segunda herramienta es la realización de una evaluación por parte de evaluadores internos (directivos de la empresa), así como de algunos compañeros previamente definidos, con el fin de obtener un análisis de la conducta del candidato en su puesto de trabajo, realizando tareas de su área de responsabilidad basadas en situaciones prácticas. Los puntos sometidos a evaluación durante esta fase son la creatividad, la coordinación, la cooperación con los compañeros, la gestión del conocimiento, el trabajo con comités, actuar en situaciones inciertas y la cooperación con otros departamentos. En función de los resultados se proponen diversos programas de desarrollo de competencias individualizados. Debido a que las evaluaciones se llevan a cabo de manera individual, el evaluador puede 
compartir sus impresiones con otro evaluador dentro de la misma situación, llamado "persona de contraste», para verificar su evaluación. Finalmente, a través de una entrevista, el candidato y el evaluador intercambian impresiones sobre su futuro rol como directivo, sobre su autorresponsabilidad mostrando motivación e interés, sobre las áreas de mejora que debe potenciar y sobre el rendimiento alcanzado en la evaluación de las situaciones prácticas reales.

El Sr. Fernández (de 51 años de edad, con más de 25 años de experiencia dirigiendo equipos en producción) relata su filosofía de liderazgo diaria: «Al personal hay que ir planteándole retos y ver los resultados, y esto solo se puede realizar en un proceso de evaluación y formación continua, la clave del éxito creo que está en que todo el personal tenga oportunidades para poder mostrar el talento que lleva dentro». La Srta. Isabel (32 años) hace referencia al programa de desarrollo individualizado: «El candidato debe potenciarse mediante programas de formación prácticos que estén destinados a desarrollarlo (habilidades, técnicas de gestión, etc.)». También el Sr. Díaz remarca: «Para potenciar el talento es necesario adaptar un programa personalizado acorde al candidato».

\subsubsection{Fase 03. Dar visibilidad y evaluar el talento}

Durante esta fase, el candidato debe llevar a cabo situaciones similares a su función como directivo y es evaluado a través de su desempeño. En este período los observadores deberán comparar la imagen que tiene el candidato acerca de sí mismo con la que tienen los demás sobre él. Es importante que en esta fase el candidato aumente su visibilidad en otras áreas, puesto que entra a formar parte del global talent pool, donde hay otros candidatos en su misma situación pertenecientes a otras áreas de la empresa. Para ello, se evalúa al candidato nuevamente, pero esta vez en situaciones similares a su rol de directivo, siendo la exigencia mayor que en las evaluaciones realizadas en la fase de desarrollo.

Dentro del marco de las entrevistas realizadas, un ejemplo es Alejandro (mánager de 31 años y 7 en la empresa), el cual expone: «El talento se detecta observando y se confirma permitiendo que otros lo observen. Se puede potenciar dando visibilidad y oportunidad a la persona».

Al final de esta fase, se realiza la «ronda de talento», donde se consideran los resultados obtenidos, así como las necesidades de la empresa, y se decide si como candidato avanza o no a la fase final. De haber una decisión negativa, el candidato no puede avanzar a la siguiente y definitiva etapa, pudiendo repetir la fase de desarrollo o de visibilidad una vez más como máximo. Es por ello que el objetivo en la «ronda de talento» es llegar a un acuerdo común acerca del desarrollo del candidato con todos los gerentes y directores del área del candidato. 


\subsubsection{Fase 04. Confirmación del talento}

Durante esta última etapa el candidato puede continuar desarrollando sus propias competencias en el contexto del trabajo a través de los cursos de formación y entrevistas feedback formales por parte de sus directores, con tal de prepararle para un puesto de dirección, corroborando su rendimiento y obtener así una confirmación final.

«El feedback es necesario e imprescindible ya que se trata de un proceso de evaluación de comportamientos y habilidades, que tiene el objetivo identificar potencial en las áreas de mejora de las personas», comenta el Sr. José Luís (expatriado con 23 años de experiencia dentro del grupo empresarial).

Diferentes observadores deben valorar el rendimiento on the job del candidato, con base en el perfil de competencias, confirmando al candidato para una posición de dirección y facilitando a la dirección del candidato las observaciones oportunas. Es responsabilidad del candidato continuar asegurando y manteniendo las conductas asociadas a su nuevo rol de director, realizando acciones de desarrollo específicas para potenciar aspectos en algún área personal.

El Sr. Iglesias (gerente experimentado en varias áreas de la empresa desde hace más de 30 años) indica la necesidad de «observar en el día a día cómo se desenvuelve en la gestión para la consecución de los objetivos».

Finalmente, los departamentos de RR. HH. y el área a la que está adscrito el candidato deben confirmar su posición como director para asegurar su nombramiento final, puesto que de lo contrario permanece en el pool (o reserva) de talento, donde se encuentran todos aquellos candidatos que han superado todas las fases previas del programa pero no disponen de un puesto de director asignado por diferentes motivos, como, por ejemplo, la prejubilación de un gerente que se alarga en el tiempo (3-12 meses) o bien reestructuraciones forzadas por la necesidad de reducción de costes por crisis económicas como la sufrida con la covid-19. En estos casos, el candidato se ve forzado a realizar algún proyecto de modo provisional durante el período previo a ocupar el puesto definitivo. 
Figura 1. Sistema de códigos y categorías del análisis cualitativo

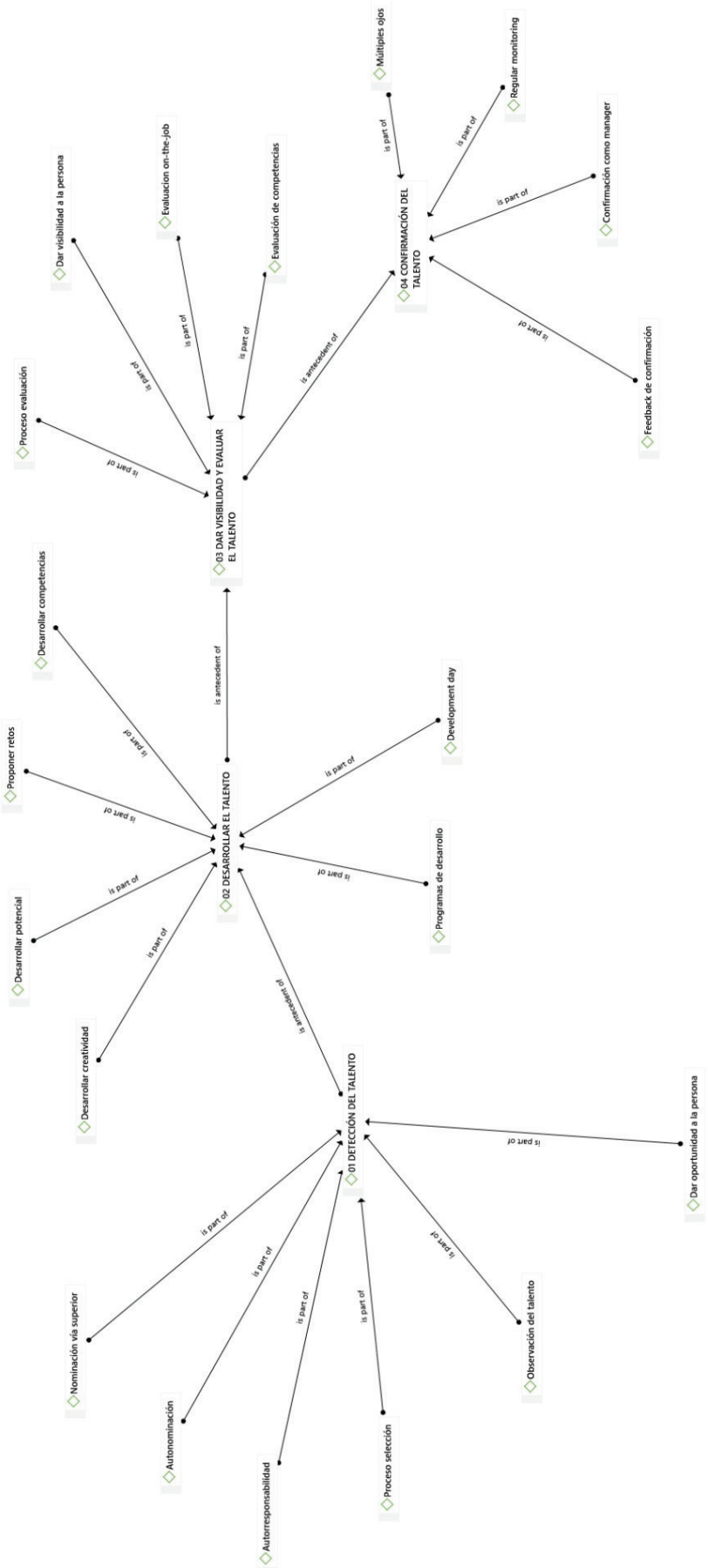




\section{Discusión sobre el programa GTM}

En el caso de estudio presentado, se observa que la empresa ha comprendido la importancia de gestionar el talento de forma global y sistemática (Tarique y Schuler, 2010), aplicando una planificación estratégica de los RR. HH., generando un pool de talento, ofreciendo múltiples vías de desarrollo profesional y fomentando de igual manera la movilidad intra- e interárea a través de observaciones por múltiples evaluadores (directivos de diversas áreas) que en un futuro puedan contar con esos candidatos evaluados.

Adentrándose en la gestión global del talento, la empresa ha definido actividades organizativas con el objetivo de atraer, seleccionar, desarrollar y retener talento para las funciones de dirección a nivel tanto nacional como internacional (Scullion et alii, 2010). Sin embargo, en la presente investigación, nos hemos centrado en el ámbito español (no la parte multinacional), cuyas fases han sido definidas como detección, desarrollo, visibilidad y evaluación, y confirmación. A través del programa GTM, se logra generar un pool de talento tanto para puestos que actualmente se encuentran vacantes como para tener una reserva de candidatos para ocupar puestos de dirección en un futuro próximo. Esta es una estrategia reconocida por autores como Mäkelä et alii (2010).

El programa GTM desarrollado por la empresa es novedoso en tres aspectos: la autonominación, la evaluación on the job y la evaluación por múltiples evaluadores. Ello puede asimilarse a las fases de identificación, diseño y evaluación del proceso de desarrollo de talento definido por Garavan et alii (2012), pero con notables diferencias. El primer aspecto diferencial que aporta el programa GTM de la empresa analizada es la potenciación de la autorresponsabilidad, ante la posibilidad de que el candidato se autonomine como talento. Como hemos observado en la revisión de la literatura, la principal fuente de talento son los propios empleados de la empresa (Tarique y Schuler, 2010), por lo que el reto de llegar a esta reserva de talento es potenciado a través de los candidatos mismos, puesto que son ellos quienes conocen sus capacidades e intereses. La retención del talento en la empresa analizada se ha convertido en una prioridad estratégica para el área de RR. HH. con el propósito de animar a los empleados a permanecer en la organización. En el caso de estudio, la empresa ha optado por permitir al empleado autonominarse como candidato al programa de GTM con la única condición del cumplimiento de requisitos. A través de esta metodología, la empresa hace frente a uno de los mayores problemas actuales en la retención del talento, que es la nominación por parte del superior jerárquico (DeVos et alii, 2007). Hay estudios que afirman que un tercio de los mejores trabajadores no se sienten a gusto con su jefe y están buscando un nuevo puesto de trabajo conti- 
nuamente (Mukhtar et alii, 2011). La autonominación es una opción que puede evitar la fuga de talento en estas situaciones. Otro aspecto disruptivo en el programa GTM analizado es la evaluación on the job del candidato, pues sustituye al sistema más común de la evaluación en un assessment center donde la evaluación se realiza y decide mediante una serie de entrevistas y role plays en un centro diferente al puesto de trabajo. En cambio, con la evaluación on the job se procede a la realización de pruebas reales donde el candidato es evaluado por el desempeño dentro de su propia área de trabajo. Con esta metodología, el candidato se somete directamente a la evaluación realizando sus funciones. La evaluación on the job también permite al candidato ampliar sus conocimientos y habilidades, lo que es fundamental para un programa de GTM (Fernández-Aráoz et alii, 2011). El último aspecto diferencial del programa GTM analizado es la evaluación por múltiples observadores. Esta característica del programa permite aumentar la fiabilidad y la transparencia del proceso de evaluación. Sin embargo, en la empresa analizada, este aspecto conlleva uno de los inconvenientes más relevantes del programa GTM, que es la disponibilidad de los evaluadores, dado que deben agregar a sus ya complicadas agendas (viajes, reuniones, formación, etc.) la asistencia a las reuniones de evaluación de los candidatos. La necesidad de los observadores de desplazarse para asistir a las entrevistas y reuniones de evaluación, unida a la escasa disponibilidad de tiempo de estos observadores, provoca frecuentes cambios y cancelaciones de última hora, lo que dificulta el desarrollo de las actividades en las fechas previstas. Como consecuencia, en ocasiones, el proceso de evaluación se puede alargar en el tiempo (hasta seis meses), generando diversas controversias entre los departamentos de los evaluadores, el de RR. HH. y el propio departamento del candidato.

\section{Conclusiones y futuras líneas de investigación}

Tras la revisión de la literatura se observa un emergente interés por la gestión del talento en las empresas como una de las principales herramientas competitivas. Los estudios académicos empiezan a combinarse con implantaciones prácticas en algunas compañías dando lugar a nuevos sistemas de gestión global del talento. El presente estudio se ha focalizado en el proceso de la gestión global de talento en una empresa del sector de la automoción, proceso que incluye las fases de detección, desarrollo, visibilidad y evaluación, y confirmación.

En el caso analizado, existen tres aspectos diferenciales en la gestión global del talento: la autonominación por parte del candidato, la evaluación on the job $y$ la evaluación por parte de múltiples observadores procedentes de otras áreas de la compañía. El primero de ellos busca la retención del talento a través de que los 
mismos empleados se autonominen para ocupar puestos de dirección, ofreciéndoles, de esta manera, una gran oportunidad para que desarrollen su carrera profesional sin abandonar la empresa. Mediante la evaluación on the job, se asegura que las competencias del candidato sean las adecuadas, puesto que desarrollará las pruebas directamente en su puesto de trabajo con objetivos y actividades que realizar dentro de su entorno diario. Finalmente, las competencias del candidato serán valoradas gracias a múltiples observadores que son directivos de la misma empresa, que, debidamente formados, presenciarán en primera persona las habilidades, actitudes y aptitudes del candidato emitiendo una valoración final.

Este estudio tiene algunas limitaciones en cuanto a la generalización de resultados, puesto que se ha analizado el proceso de gestión global del talento en el contexto de una única empresa del sector de la automoción y los resultados obtenidos pueden no ser directamente generalizables a otras compañías o sectores. Sin embargo, la divulgación de este estudio puede resultar útil en otras empresas de dentro y fuera del sector industrial, a nivel tanto nacional como internacional.

En cuanto a líneas futuras de investigación, la gestión global del talento es un campo puente, dado que es a la vez teórico y práctico (Tarique y Schuler, 2010). Es necesario que tanto los académicos como los profesionales de la gestión del talento compartan sus intereses y desarrollen proyectos de investigación conjuntos que permitan alcanzar los objetivos e intereses de ambos colectivos. Se necesitan más trabajos de investigación que faciliten la transferencia de conocimiento de los académicos a los profesionales y viceversa. Un área de investigación futura es la del diseño y configuración de los sistemas de gestión global del talento, y cómo los sistemas de gestión global del talento repercuten en la eficacia de la organización (Tarique y Schuler, 2010). Adicionalmente, la fase de selección de talento puede ser una vía de investigación futura, como complemento a las fases analizadas en el presente estudio. ¿Cómo son y cómo deberían ser los procesos de búsqueda, selección e identificación del talento para lograr una mayor competitividad en las empresas? En este sentido, el programa GTM expuesto en este estudio abre el campo a la retención del talento a través de la autonominación por parte de los candidatos que, por ejemplo, no tengan una buena relación con su superior jerárquico. Futuras investigaciones podrían profundizar en las ventajas e inconvenientes de la autonominación como vía para acceder al programa de gestión del talento frente a la nominación por parte del superior jerárquico o a la búsqueda externa de talento mediante head bunting. Paralelamente a todo lo expuesto, estudios sobre el liderazgo de las nuevas generaciones han puesto de manifiesto que las preferencias de desarrollo profesional difieren respecto a generaciones anteriores (Gallardo, 2016), y que estas no se limitan únicamen- 
te a unas mejores condiciones laborales, sino que aparecen inquietudes y motivaciones nuevas, como, por ejemplo, liderar proyectos con objetivos ambiciosos independientemente del nivel jerárquico que uno tenga. Este hecho abre una vía de investigación futura sobre la gestión del talento en las nuevas generaciones de empleados, ya que ello puede suponer un desafío mayor en comparación con los empleados de generaciones anteriores, los cuales tenían unos principios basados en los incentivos económicos y el ascenso jerárquico en la compañía (Gallardo, 2016). Finalmente, cabe señalar que, debido al contexto motivado por la pandemia global de la covid-19, las estrategias de digitalización y telecomunicación han acelerado la implementación de nuevos procesos y herramientas digitales en muchas compañías, por lo que resultaría interesante adentrarse en el estudio de los factores asociados a la digitalización y el teletrabajo que están influyendo o pueden influir en la gestión global del talento.

\section{Bibliografía}

Arkless, D. et alii (2011). «Global Talent Risk, Seven Responses», Foro Económico Mundial y el Boston Consulting Group.

Björkman, I., Ehrnrooth, M., Mäkelä, K., Smale, A. y Sumelius, J. (2013). «Talent or Not? Employee reactions to talent identification». Human Resource Management, 52 (2), 195-214.

Bonneton, D., Festing, M., Schworm, S. K. y Muratbekova-Touron, M. (2019). «Do global talent management programs help to retain talent? A career-related framework». The International Journal of Human Resource Management. Disponible en: <https://doi.org/10.1080/09585192.2019.16 $83048>$

Boudreau, J. W. y Ramstad, P. M. (2005). «Where's Your Pivotal Talent?» Harvard Business Review, 83, 23-24.

Bradberry, T. (2016). "Ocho errores que hacen que los mejores trabajadores dejen su puesto». Huffpost. Disponible en: http://www.huffingtonpost.es/drtravis-bradberry/errores-trabajadores-dimiten_b_9758820.html

Byars, L. L. y Rue, L. W. (1994). Human Resources Management (4. a ed.). Burr Ridge, IL: Irwin.

Caligiuri, P. y Tarique, I. (2009). «Predicting effectiveness in global leadership activities»+ Journal of World Business, 44 (3), 336.

Cheese, P. (2010). «Talent management for a new era: what we have learned from the recession and what we need to focus on next». Human Resource Management International Digest, 18 (3), 3-5. 
Chen J. H., Lee T. L. y Ting I. H. (2017). «A Study of Headhunter's Recruiting on Social Network Sites: Take LinkedIn as an Example». Proceedings of the $4^{\text {th }}$ Multidisciplinary International Social Networks Conference.

Collings, D. G. y Mellahi, K. (2009), «Strategic talent management: A review and research agenda». Human Resource Management Review, 19 (4), 304.

Collis, J. y Hussey, R. (2009). Business Research: A Practical Guide for Undergraduate and Postgraduate Students (3. ${ }^{\mathrm{a}}$ ed.). Basingstoke: Palgrave Macmillan.

Colombo, D. (2006). Señales de Talento. Daniel Colombo Coaching, comunicación, motivación y liderazgo. Disponible en: <http://danielcolombo.com/ senales-como-darte-cuenta-si-alguien-tiene-talento-profesional>

Crossley, M. (2007). "Narrative analysis. Analysing qualitative data in psychology», en E. Lyons y A. Coyle (eds.). Analyzing Qualitative data in Psychology (pp.131-144). London: Sage.

Crowley, M., Benson, E. y Akram, A. (2019). «Linking Talent Management to Traditional and Boundaryless Career Orientations: Research Propositions and Future Directions». European Management Review, 16, 5-19.

DeVos, A. y Meganck, A. (2007). «What HR Managers do versus what employee's value: Exploring both parties' views on Retention Management from a Psychological contract perspective». Personel Review, 38 (1), 45-60.

Eisenhardt, K. M. (1989). Building theories from case-study research. Academy of Management Review, 14 (4), 532-550.

Eisenhardt, K. M. y Graebner, M. E. (2007). «Theory building from cases: opportunities and challenges». Academy of Management Journal, 50 (1), 2532.

Farndale, E., Scullion, H. y Sparrow (2010). «The role of the corporate HR function in global talent management». Journal of World Business, 45 (2), $161-168$.

Fernández-Aráoz, C., Groysberg, B. y Nohria, N. (2011)+ «How to hang on to your high potentials». Harvard Business Review, 89 (10), 76-83.

Frauenheim, E. (2009). «Talent planning for the times». Workforce Management, 88 (11), 37-39, 41-43.

Freedman, E. (2009). «Optimizing Workforce Planning Processes»+ People E Strategy, 32 (3), 9-10.

Gallardo, E. (2016). «Standing on the shoulders of giants? A critical review of empirical talent management research». Employee Relations, 38 (1), 31-56.

Gallardo, E., Dries, N. y González-Cruz, T. (2013). «What meaning talent world work». Human Resource Management Review, 23 (4), 290-300. 
Garavan, T.N., Carbery, R. y Rock, A. (2012). «Mapping talent development: definition, scope and architecture». European Journal of Training and Development, 36 (1), 5-24.

Gibiert, M. y Ruigrok, W. (2010). «The 'what' and 'how' of case study rigor: three strategies based on published work». Organizational Research Methods, 13 (4), 710-737.

Givhan, R.N.(2014).Developing next generation Leaders in a diverse environment. Bloomington: Author House TM.

Goldstein, I. L. (1990). «Training in work organizations». En M. D. Dunutte y L. M. Hough (eds.). Handbook of Industrial E organizational Psychology (pp. 507-619). Palo Alto, CA: Consulting Psychologists Press.

Guest, G., Bunce, A. y Johnson, L. (2006). «How many interviews are enough? An experiment with data saturation and variability.» Field Methods, 18 (1), 59-82.

Guthridge, M., McPherson, J. R. y Wolf, W. J. (2009). «Upgrading talent». The McKinsey Quarterly, (1), 61.

Harttig, M. A. (2010). «Global Workforce Planning». Benefits E Compensation International, 40 (1), 19.

Hills, A. (2009). «Succession planning - or smart talent management?» Industrial \& Commercial Training, 41 (1), 3-8.

Huselid, M. A. (1995). «The impact of human resource management practices on turnover, productivity, and corporate financial performance». Academy of Management Journal, 38, 635-672.

Iles, P., Chuai, X. y Preece, D. (2010). «Talent Management and HRM in Multinational companies in Beijing: Definitions, differences and drivers». Journal of World Business, 45, 179-189.

Latham, G. P., Saari, L. M., Pursell, E. D. y Campion, M. (1980). «The situational interview». Journal of Applied Psychology, 65, 422-427.

Lawler, E. E. (2008). Talent: Making people your competitive advantage. San Francisco, CA US: Jossey-Bass.

Lewis, R. E. y Heckman, R. J. (2006). «Talent management: A critical review». Human Resource Management Review, 16 (2), 139.

Mäkelä, K., Björkman, I. y Ehrnrooth, M. (2010). «How do MNCs establish their talent pools? Influences on individuals' likelihood of being labeled as talent». Journal of World Business, 45, 134-142.

McDonnell, A., Lamare, R., Gunnigle, P.y Lavelle, J. (2010). «Developing tomorrow's leaders: Evidence of global talent management in multinational enterprises». Journal of World Business, 45, 150-160. 
Michaels, E., Handfield-Jones, H. y Axelrod, B. (2001). War for Talent. Boston: Harvard Business School Press Books.

Miles, M. B. y Huberman, A. M. (1994). Qualitative data analysis: An expanded sourcebook. Beverly Hills: Sage Publications.

Mukhtar, U., Siengthai, S. y Ramzan, S. (2011). «Mediating Role of HRM in organizational Conflict and Impact on Organizational Effectiveness: Empirical Evidence of Pakistan Public Universities». International Journal of Business, Management and Economic Research, 2 (6), 391-403.

NaIm, M. F. (2014). «Leveraging Social Media for Generation Y retention». European Journal of Business and Management, 6 (23), 173-180.

Nijs, S., Gallardo, E., Dries, N. y Sels, L. (2014). «A multidisciplinary review of talent». Journal of World Business, 49, 180-191.

Riessman, C. K. (2005). «Narrative Analysis». En N. Kelly et alii (eds.). Narrative, Memory \& Everyday Life. Huddersfield: University of Huddersfield.

Rynes, S., Gyluk, T.y Brown, K. (2007). «The very separate worlds of academic and practitioner periodicals in human resource management: Implications for evidence-based management». Academy of Management Journal, 50, 987 1008.

Schweyer, A. (2004). Talent management systems: Best practices in technology solutions for recruitment, retention and workforce planning. New Jersey: John Wiley \& Sons.

Scullion, H., Collings, D. G. y Caligiuri, P. (2010). "Global talent management». Journal of World Business, 45 (2), 105-108.

Sheridan, J. E. (1992). «Organizational culture and employee retention». Academy of Management Journal, 35 (5), 1036-1056.

Stahl, G., Bjökman, I., Farndale, E., Morris, S., Paauwe, J., Stiles, P., Trevor, J. y Wright, P. (2007). Global Talent Management: How Leading Multinationals Build and Sustain Their Talent Pipeline. Fontainebleau, France: INSEAD.

Stahl, G., Bjökman, I., Farndale, E., Morris, S., Paauwe, J., Stiles, P. y Wright, P. (2012). «Six principles of effective global talent management». Sloan Management Review, 53 (2), 25-42.

Sylvester, J. (2004). «Creating and maintaining an engaged Generation Y workforce». Emerald Group Publishing Limited, 14 (4).

TAnsey, O. (2007). «Process tracing and elite interviewing: A case for nonprobability sampling». Political Science and Politics, 40 (4), 765-772. 
TAnsley, C. (2011). «What do we mean by the term“Talent' in talent management? Industrial and Commercial Training», Industrial and Commercial Training, 43 (5), 266-274.

Tansley, C., Turner, P. y Foster, C. (2007). Talent: Strategy, management, measurement: Research into practice. London: CIPD.

Tarique, I. y Schuler, R. S. (2010). «Global Talent Management: Literature Review, Integrative Framework, and Suggestions for Further Research». Journal of World Business, 45 (2), 122-133.

Vasile, G. y Zhan, X. (2020). «Recruitment, Selection and Integration in the Human Resource Management». Internal Auditing E Risk Management, 34.

Williams, M. (2000). The war for talent: Getting the best from the best. London: CIPD.

Xuy, H., Yuy, Z., Yangz, J., Xiongz, H. y Zhu, H. (2016). Talent Circle Detection in Job Transition Networks. ACM. Disponible en: <http://dx.doi. org/10.1145/2939672.2939732>

YIN, R. K. (2011). Applications of case study research. Los Angeles, California: Sage. 